\title{
The surgical management of morbidly obese women with endometrial cancer
}

\author{
Stephanie Kuku ${ }^{1}$ John Dick ${ }^{2}$, Adeola Olaitan $^{1^{*}}$ \\ ${ }^{1}$ Department of Gynaecologic Oncology, University College London Hospitals, London, UK; \\ *Corresponding Author: adeola.olaitan@uclh.nhs.uk, stephanie.kuku@uclh.nhs.uk \\ ${ }^{2}$ University College London Hospitals, London, UK; John.Dick@uclh.nhs.uk
}

Received 5 November 2012; revised 8 December 2012; accepted 20 December 2012

\begin{abstract}
There is an obesity epidemic in the Western World. Currently one in four adults is obese and three in ten children in the UK is overweight or obese [1]. The UK Foresight Study [2] predicts that if nothing is done, this figure will rise to five in ten $(50 \%)$ in women by 2050 . The endocrine changes induced by obesity lead to an increased incidence of endometrial cancer. The majority of women with endometrial cancer present with early disease which means that surgery will form all or part of their treatment. This presents serious challenges as these women often have associated co-morbid conditions. In this article we explore the relationship between obesity and endometrial cancer and highlight the surgical challenges.
\end{abstract}

Keywords: Obesity; Endometrial Cancer; Laparoscopic Surgery; Vaginal Surgery; Robotic Surgery

\section{INTRODUCTION}

The rising rates of obesity globally continue to have significant implications for health and health economics. Having a body mass index in the overweight or obese range is the fifth leading cause of death worldwide, accounting for over 2.8 million deaths every year [3]. In England, just over a quarter of adults ( $26 \%$ of both women and men aged 16 or over) are obese. In 2010, around three in ten boys and girls (aged 2 to 15) were classed as either overweight or obese [1]. The World Health Organization (WHO) [4] defines normal body weight as a body mass Index (BMI) of 18 to $24.9 \mathrm{~kg} / \mathrm{m}^{2}$, overweight, BMI of 25 to $29.9 \mathrm{~kg} / \mathrm{m}^{2}$, obesity, BMI $\geq 30.0 \mathrm{~kg} / \mathrm{m}^{2}$ ), and morbid obesity, BMI $\geq 40 \mathrm{~kg} / \mathrm{m}^{2}$. US data has showed an increase in obesity in the heaviest women with a $75 \%$ increase in women with a BMI $>50 \mathrm{~kg} / \mathrm{m}^{2}$ and a $52 \%$ increase in women with BMI > 40, compared with a $24 \%$ increase in women with BMI $>30$ [5]. Clinical guidelines backed by strong evidence will be needed to guide management of cancer in these women if these figures continue to rise as predicted.

The relationship between cancer and obesity is wellestablished, and obesity is related to a 2-5-fold risk of endometrial cancer, the highest risk association for any cancer. Morbidly obese women have a nine times increase risk compared to women of normal BMI [6] and this group of women with endometrial cancer and a BMI $\geq 40$ have the highest relative risk of death for any morbidity related cancer [7].

Endometrial cancer is the fourth most common female cancer in the UK, accounting for $5 \%$ of cancers in women, with $93 \%$ of cases in women over the age of 50 . There were 7703 new diagnoses in 2008, with a $40 \%$ rise in the last 15 years [8], making it the commonest gynaecological cancer in women. The most important risk factors for endometrial cancer are unopposed oestrogen exposure, endometrial hyperplasia and obesity [9]. The hypothesis for development of endometrial cancer in obese women is thought to be through a hormone-dependent pathway ("type I endometrial cancer") relative to thin women who may develop disease through autonomous oncogenesis ("type II") $[10,11]$. Most endometrial cancers are type I, early stage, with a good prognosis. Over $80 \%$ of patients will present with early-stage (FIGO 2008 Stage I) disease and five-year survival is over $85 \%$. Estimates suggest that up to $90 \%$ of women with Type I cancer patients are obese [12]. Traditionally, standard primary treatment for early endometrial cancer is surgical; total abdominal hysterectomy (TAH) and bilateral salpingo-oopherectomy. In selected cases there may be a case for pelvic and para-aortic lymphadenectomy though there is no evidence to support routine lymph-node dissection in early-stage disease [13]. In the last decade, primary surgery and staging have been performed more often with laparoscopy [14-22] as a safe, effective alternative, with fewer operative complications and more rapid recovery. 
This is of even greater importance for obese and morbidly obese women who pose medical, anaesthetic, and surgical challenges. Randomised controlled trials and large retrospective studies have shown laparosopic hysterectomy (TLH) and more recently, robotic surgery (total robotic hysterectomy, TRH) to be both feasible, safe and effective in these high-risk obese and morbidly obese women.

\section{MORBID OBESITY-MANAGEMENT}

\subsection{Patient Work-Up}

Morbid obesity carries an increased risk of complications and mortality especially during and after surgery. This group of women present added challenges to surgeons as they usually suffer from obesity-driven comorbidities such as type II diabetes, hypertension, heart disease, osteoarthritis and pulmonary disease [23]. The difficulties usually encountered during surgery in this patient group and the high risk of perioperative complications warrant a full medical and anaesthetic pre-operative assessment to identify and manage any existing medical problems. Having a BMI $>40$ greatly influences decisions regarding treatment modalities depending on the number of co-morbidities, perceived anaesthetic risk, and experience of the surgeon. As conversion rates for minimally invasive procedures tend to be higher in this group, choosing the right type of surgery from the outset will reduce conversion rates and risk of complications. Palomba et al. [24] were unable to identify predictors of conversion using intravesical fat (IVC) assessments obtained from initial staging CT and US in 151 obese women. A full clinical and pelvic examination will help the surgeon to adequately plan surgery, preempt difficulties with (vaginal) access, uterine size, and plan incisions.

\subsection{Surgery for the Morbidly Obese-Evidence for Laparoscopic Approach}

It is now generally accepted that the laparoscopic approach (TLH) to surgery in both non-obese and obese women with endometrial cancer can have advantages over traditional open hysterectomy with fewer minor and major complications, less blood loss, shorter hospital stay and similar survival rates. TLH has also been shown to be quicker, more efficient and with less blood loss than laparoscopically-assisted vaginal hysterectomy (LAVH) [25].

Randomized controlled trials provide evidence that patients undergoing minimally invasive surgery can have better outcomes than those who have a laparotomy. These trials only include a small proportion of obese and morbidly obese women. The data for morbidly obese women alone is limited. In the Gynecologic Oncology Group LAP-2 study [22], 2616 patients were randomly assigned to laparoscopy or laparotomy. Laparoscopy resulted in fewer postoperative moderate or severe complications, shorter hospital stay, but with longer operative times. All patients had complete surgical staging including pelvic and para-aortic lymph node dissection. Conversion to laparotomy rate in the laparoscopy group was $26 \%$ in this study, with a clear relationship between increasing BMI and rate of conversion to laparotomy.

Walker et al. in Australian LACE study [21] of 332 women also demonstrated fewer moderate to severe complications, and shorter hospital stays. Patients who had a TLH also reported greater improvement in quality of life (QoL) from baseline compared to those who had TAH. $41(23.2 \%)$ women in the TLH group and $26(19.3 \%)$ in the TAH group were classed as morbidly obese. Postoperatively, twice as many patients in the TAH group experienced grade 3 complications. Conversion rate was 2.4\% -7 patients from TLH to TAH and one from TAH to TLH (patient choice).

Mourtis et al. [20] published data from a Dutch multicenter randomized controlled trial showing total laparoscopic hysterectomy (TLH) to be associated with lower morbidity particularly in obese women. 238 women were randomly allocated to either TLH or TAH. Median BMI in the TLH group was $29 \mathrm{~kg} / \mathrm{m}^{2} ; 41 \%(\mathrm{n}=117)$ of patients in this study had a BMI of $>30$. Conversion rate in the TLH group was $10.8 \%$. TLH was found to be significantly associated with less blood loss, less use of pain medication, a shorter hospital stay, faster recovery, and longer operating.

A UK based retrospective study [26] of 191 obese and morbidly obese women with endometrial cancer illustrated low conversion rates (1\%) and complications rates in a cohort of patients who underwent TLH. 53/191 (28\%) of women were classed morbidly obese, and $23(12 \%)$ class II obese (BMI 35 - 39.9). All women with high BMI were treated preferentially with laparoscopy (TLH) over the period reviewed (2003-2009). There was a small increase in median operating time for obese compared to normal weight women, however this was not observed for the morbidly obese group. There were no significant differences in either intra-operative or post-operative complications between normal, obese, and morbidly obese women. This is the largest cohort in the UK to provide evidence of feasibility and safety of laparoscopic surgery in morbidly obese women with endometrial cancer.

O'Hanlan et al. [27] analysed retrospective data of 90 patients having TLH for uterine pathology over 9 years, stratifying by BMI (range 18 to $60 \mathrm{~kg} / \mathrm{m}^{2}$ ), with 12 morbidly obese patients with BMI between 40 and 61 . There were no significant associations found between complications, conversion to laparotomy and BMI. Yu et al. [28] 
evaluated the feasibility of TLH as the primary treatment for endometrial cancer in eight morbidly obese women with a mean BMI (44.8) in TAH group, and (45) in TLH group. They found equivalent operating times, without evidence of greater operative morbidity in the laparoscopy group. There were no conversions to laparotomy. Median length of hospital stay was shorter in the TLH group (4 vs 11.4 days). Scribner et al. [29] retrospectively analysed 356 obese patients, $22 \%$ of which were classed as morbidly obese; these women had a significantly higher incidence of diabetes, hypertension and pulmonary disease. Women with BMI $>40$ were significantly younger then those with BMI $<30$ (58 vs 65 years, $\mathrm{p}=0.001$ ). Women in the TLH group had longer operating times, shorter hospital stays, as well as fewer post operative complications. This study found a positive association between increasing BMI and conversion from TLH to TAH. Other [30,31] retrospective studies which include obese and morbidly obese women show that endometrial cancer can be safely managed with laparoscopic surgery in these women.

Laparoscopic treatment remains an effective, safe modality for the morbidly obese. The number of gynaecologic oncologists trained in laparoscopic surgery continues to rise. It has been suggested that conversion rates in patients with high BMI can be overcome as more surgeons become proficient in the technique and receive more experience in operating on morbidly obese patients. Unpublished data from a retrospective review in our center of a cohort of obese $(n=80)$ and morbidly obese $(n=$ 53) revealed low complication rates with a conversion rate of $4.5 \%$ (6/133). Median BMI in the morbidly obese group was 48 (range 40 - 63). The evidence suggests that the laparoscopic approach should be the standard of care for obese women requiring surgery for endometrial cancer.

\subsection{Anaesthetic Considerations}

Laparoscopy necessitates a general anaesthetic and positive pressure ventilation of the lungs in all patients to offset the reduced diaphragmatic compliance of the pneumoperitoneum. An excellent generic guideline for perioperative management of the morbidly obese has been produced by the Association of Anaesthetists of Great Britain and Ireland. (Ref.

http://www.aagbi.org/sites/default/files/Obesity07.pdf The importance of identifying sleep apnoea during pre-assessment must be appreciated as this will guide provision of continuous positive airway pressure (CPAP) peri-operatively, and may be associated with pulmonary hypertension and right heart failure. Practical considerations include how to safely transfer the patient on and off a suitable operating table. Transfers can be minimized by the patient positioning themselves on the operating table prior to induction of anaesthesia, and by the use of a suitable device such as a hover mattress for transfer onto postoperative bed at the end of the case. Invasive arterial pressure monitoring is usually required as blood pressure cuffs read inaccurately on the obese arm.

Airway management includes pre-medication with a proton pump inhibitor to reduce likelihood of aspiration. Obese patients have a reduced oxygen reserve (due to decreased functional residual capacity (FRC) and increased tissue oxygen demand) and may be harder to intubate. It is advised to use the ramped position for intubation to increase FRC and oxygen reserve and to make ventilation easier. In cases of predicted airway difficulties an awake fibreoptic intubation may be indicated before inducing anaesthesia. Gastric drain insertion under anaesthesia with a wide bore tube (orogastric preferable) reduces gastric distension secondary to mask ventilation thus allowing safer introduction of a laparoscopic port in the left hypogastrium (Palmer's point), and reduces aspiration risk. A nationwide audit of all anaesthesia-related airway complications resulting in either death, brain damage, emergency tracheostomies or unintended ICU admission found a doubling of risk in the obese population (4th National Audit Project of the Royal College of Anaesthetists, Major complications of airway management in the UK.

http://www.rcoa.ac.uk/document-store/nap4-full-report).

The physiological challenges associated with a carbon dioxide pneumoperitoneum include; hypercapnia, decreased FRC and lung compliance (exacerbated by head down tilt required for exposure of pelvic organs) leading to relative hypoventilation, high lung inflation pressures leading to increased TPR and PAP, a reduction in venous return and cardiac output, and increased regurgitation risk [32]. The anaesthetic technique often involves tolerating hypercapnia and relative hypoxaemia intraoperatively, and recruitment techniques after closure of the pneumopertioneum. It may be necessary to assist ventilation postoperatively in the head-up positon on the critical care unit (CCU) for a short period before extubation. Another indication for CCU admission is facial and airway oedema resulting from prolonged head-down positioning which may also require a period of assisted ventilation.

There is little evidence to quantify perioperative risk in this specific patient population but in our unit [33], we have found that there were no major complications in our case series, but an increased rate of postoperative ventilation in those with a BMI $>50$.

\subsection{Vaginal Hysterectomy}

In morbidly obese women with co-morbidities such as extensive intra-peritoneal scarring, chronic respiratory disease or cardiac compromise, laparoscopy may be contraindicated. Vaginal hysterectomy has been advocated as 
a suitable alternative in these women undergoing hysterectomy for benign disease [34-36]. Some argue that staging is inadequate as this route does not allow for exploration of the abdominal cavity, peritoneal washings, and lymph-node dissection if indicated. Although vaginal hysterectomy can be difficult or impossible to perform in women with very high BMI with poor access (especially in nulliparous), it remains an adequate and feasible option in some [37]. Guigale et al. [38] show feasibility and outcome for morbidly obese women with endometrial cancer treated with vaginal hysterectomy (grouped with TLH, and robotic under minimally invasive surgery (MIS), and compared to TAH). Our institution conducted a retrospective audit which identified ten women with BMI > 40 treated for stage I endometrial cancer with uncomplicated vaginal hysterectomies. All ten women had significant comorbidities (1 woman had an episode of bronchospasm at anaesthetic induction and did not undergo a TLH as planned, whilst another (BMI-46) was converted to $\mathrm{VH}$ due to dense adhesions in the pelvis and restricted visibility). Two (2) women had significant aortic stenosis and previous laparotomies, one was wheelchair bound due to osteoarthritis, whilst another was considered too great an anaesthetic risk with a BMI > 50 and severe COPD).

\subsection{Robotic Surgery}

Laparoscopy remains a universally accepted and practiced surgical technique for performing a hysterectomy in women with endometrial cancer, however detractors against this approach point to the steep learning curve, concerns with inadequate staging in women requiring lymphadenectomy, and prolonged operating times. As the prevalence of obese and morbidly obese women continues to rise, gynecologists continue to try to identify and practice new surgical techniques that will enable optimal staging and treatment as well as minimise peri-operative morbidity and mortality in these patients.

Robotic-assisted surgical staging and treatment, a relatively new technique in gynecological oncology practice, has been suggested as an alternative for open surgery with some advantages over laparoscopic staging. Da Vinci first received approval from the US Food and Drug Administration (FDA), for the robotic operating system in 2005. Feasible in both obese and morbidly obese women, there may also be predictors of early conversion that can be performed preoperatively. The benefits of robotic technology over conventional laparoscopy include; working in and viewing a 3D image, increased degree of freedom, as well as improved ergonomics for the surgeon.

Gehrig et al. in their study[39], published data for 36 obese and 13 morbidly obese women who underwent surgery with the DaVinci robotic system and 25 obese and 7 morbidly obese women who underwent traditional laparoscopy, for endometrial cancer staging For both the obese and morbidly obese women, they showed that robotic surgery was superior to conventional laparoscopy with shorter operating time $(\mathrm{p}=0.0004)$, less blood loss $(p<0.0001)$, increased lymph node retrieval $(p=0.004)$ and shorter hospital stay $(\mathrm{p}=0.0119)$. There were 2 conversions to laparotomy in the TLH group and none reported in the TRH cohort. Tang and colleagues [40] published data from a retrospective cohort of 129 obese patients (mean BMI 39.8) who underwent robotic staging, compared to 110 women (mean BMI 40.3) who had a laparotomy, showing robotic staging was feasible in obese women with fewer abdominal wound, but more vaginal cuff complications.

In a large retrospective study in obese and morbidly obese women with endometrial cancer comparing all the treatment options discussed, Giugale et al. [38] analysed 659 obese patients (57.5\% (379); TAH and $42.5 \%$ (280)); minimally invasive surgery (included TLH, vaginal hysterectomy (TVH) and robotic hysterectomy (TRH)). Mean BMI was $43 \mathrm{~kg} / \mathrm{m}^{2}$, and women were grouped based on BMI: 39.6\% (261) were obese (BMI 30 - 39), 41\% (259) of the women were morbidly obese (BMI 40 - 49), with $18.7 \%$ (123) classified as super obese (BMI >= 50). Minimally invasive procedures were attempted in 280 of all 659 patients with a conversion rate of $16.6 \%$; BMI was higher in the converted group (BMI 47.3 vs 40.6; p $<0.001)$. They showed that morbidly obese women with BMI $>40$ have worse surgical outcomes than less obese women, regardless of type of hysterectomy. As obesity increased, there was a decreased frequency of lymphadenectomy, increased blood loss and fewer lymph nodes removed. Multivariate analysis showed an association with increasing BMI and wound complications $(\mathrm{p}<0.001)$ and the presence of post-operative complications $(\mathrm{p}=$ 0.003 ). Blood transfusion, hospital readmission and postoperative ileus were significantly associated with open procedures but not with obesity. This study also showed that robotic hysterectomy for all obesity groups was associated with an increased frequency of post-operative ventilation $(6.5 \%)$ compared to open and laparoscopic surgery. This finding warrants questions about anaesthetic challenges that might be associated with this new procedure in high BMI groups. Other studies [41,42] comparing robotic hysterectomy to open procedures in obese women with endometrial cancer confirm the feasibility in this patient group. Prospective trials are needed to further explore the future of robotic surgery in this high risk patient group.

\subsection{Post-Operative Care}

A validated post-operative pathway should be implemented in all units involved in the care of morbidly obese women who are at great risk of post-operative complications. Prevention and early identification of potential 
problems will reduce morbidity and mortality in these women who are at high risk of post-operative infections (wound, chest, and generalized sepsis) and thrombosis. Post-operative antibiotic prophylaxis and anti-coagulation is required in all women. The use of compression stockings, foot impulse devices or intermittent pneumatic compression devices from admission and until the patient is mobile, is recommended by the National Institute for Clinical Excellence (NICE, clinical guideline 92). Wound healing can be a problem especially as a high proportion of these women suffer from diabetes and other comorbidities, however minimally invasive surgical techniques have been shown to reduce the incidence of wound complications. Early mobilization, removal of Foley catheter and introduction of a regular diet will encourage quick recovery and prevent paralytic ileus. High-dependency care may be required initially after surgery to stabilise women who prove to be of greater anaesthetic challenge.

\section{CONCLUSION}

As the incidence of morbid obesity and endometrial cancer both continue to rise in the UK and the rest of the western world is it important that all gynaecological oncologists are trained and experienced in laparoscopic surgery in the morbidly obese. Vaginal hysterectomy, if feasible is a safe alternative in patients in whom laparoscopy is contraindicated. The uptake of robotic surgery is expected to rise as more centers around the world train surgeons in this technique. Randomized controlled trials are needed to confirm that this treatment modality is also safe and effective in treatment of endometrial cancer and may even overcome some of the surgical and anesthetic challenges faced by surgeons treating morbidly obese women with endometrial cancer with laparoscopic surgery.

\section{REFERENCES}

[1] Office for National Statistics (2010) England.

[2] Foresight Project (2007) Tackling obesities: Future Choices Foresight Project. http://foresight.gov.uk

[3] Branca, F., Nigogosian, H. and Lobstein, T. (2007) The challenge of obesity in the WHO European Region and the strategies for the response. WHO, Copenhagen.

[4] World Health Organization (2011) Obesity and overweight. http://who.int/mediacentre/factsheet/fs311/en/

[5] Sturm, R. (2007) Increases in morbid obesity in the USA: 2000-2005. Public Health, 121, 492-496. doi:10.1016/j.puhe.2007.01.006

[6] Parazzini, F., LaVecchia, C., Bocciolone, L., et al. (1991) The epidemiology of endometrial cancer. Gynecologic Oncology, 41, 1-16. doi:10.1016/0090-8258(91)90246-2

[7] Calle, E.E., Rodriguez, C., Walker-Thurmond, K. and Thun, M.J. (2003) Overweight, obesity, and mortality from can- cer in a prospectively studied cohort of US adults. The New England Journal of Medicine, 348, 1625-1638. doi:10.1056/NEJMoa021423

[8] Cancer Research UK Statistics (2008) Cancer statistics registrations: Registrations of cancers diagnosed in 2008. www.cancerresearchuk.ork

[9] Schouten, L.J., Goldbohm, R.A. and van den Brandt, P.A. (2004) Anthropometry, physical activity, and endometrial cancer risk: Results from the Netherlands Cohort Study. Journal of the National Cancer Institute, 96, 1635-1638. doi:10.1093/jnci/djh291

[10] Bokhman, J.V. (1983) Two pathogenic types of endometrial carcinoma. Gynecologic Oncology, 15, 10-17. doi:10.1016/0090-8258(83)90111-7

[11] Corocleanu, M. (1993) Hypothesis for endometrial carcinoma carcinogenesis. Preventive prospects. Clinical \& Experimental Obstetrics \& Gynecology, 20, 254-258.

[12] Von Gruenigen, V.E., Gil, K.M., Frasure, H.E., Jenison, E.J. and Hopkins, M.P. (2005) The impact of obesity and ago on quality of life in gynecologic surgery. American Journal of Obstetrics \& Gynecology, 193, 1369-1375. doi:10.1016/j.ajog.2005.03.038

[13] Kitchener, H., Swart, A.M., Qian, Q., Amos, C., Parmar, M.K. (2009) Efficacy of systematic pelvic lymphadenectomy in endometrial cancer (MRC ASTEC): A randomized study. Lancet, 373, 125-136. doi:10.1016/S0140-6736(08)61766-3

[14] De la Orden, S.G., Reza, M., Blasco, J.A., Andradas, E., Callejo, D. and Perez, T. (2008) Laparoscopic hysterictomy in the treatment of endometrial cancer: A systematic review. Journal of Minimally Invasive Gynecology, 15, 395-401. doi:10.1016/j.jmig.2008.04.018

[15] Palomba, S., Falbo, A., Mocciaro, R., Russo, T. and Zullo, F. (2009) Laparoscopic treatment for endometrial cancer: A meta-analysis of randomized controlled trials (RCTs). Gynecologic Oncology, 112, 415-421. doi:10.1016/j.ygyno.2008.09.014

[16] Palomba, S., Falbo, A., Russo, T. and Zullo, F. (2009) Updating a recent meta-analysis of randomized controlled trials to assess safety and efficacy of laparoscopic surgery for treating early stage endometrial cancer. Gynecologic Oncology, 114, 135-136. doi:10.1016/j.ygyno.2009.03.025

[17] Tozzi, R., Malur, S., Koehler, C., et al. (2005) Laparoscopy versus laparotomy in endometrial cancer: First analysis of survival of a randomized prospective study. JMIG, 12, 130-136.

[18] Palomba, S., Ghezzi, F., Falbo, A., et al. (2012) Laparoscopic versus abdominal approach to endometrial cancer; a 10 year retrospective multicenter analysis. International Journal of Gynecological Cancer, 22, 425-433.

[19] Zullo, F., Falbo, A. and Palomba, S. (2012) Safety of laparoscopy versus laparotomy in the surgical staging of endometrial cancer: A systematic review and meta-analysis of randomized controlled trials. American Journal of Obstetrics \& Gynecology, 207, 94-100. doi:10.1016/j.ajog.2012.01.010

[20] Mourtis, M., Bijien, C.B., Arts, H.J., ter Brugge, H., et al. 
(2010) Safety of laparoscopy versus laparotomy in earlystage endometrial cancer: A randomized trial. The Lancet Oncology, 11, 763-771. doi:10.1016/S1470-2045(10)70143-1

[21] Janda, M., Gebski, V., Brand, A., et al. (2010) Quality of life after total laparoscopic hysterectomy versus total abdominal hysterectomy for stage I endometrial cancer (LACE): A randomized trial. The Lancet Oncology, 11, 772780. doi:10.1016/S1470-2045(10)70145-5

[22] Walker, J.L., Piedmonte, M.R., Spirtos, N.M., et al. (2009) Laparoscopy compared with laparotomy for comprehensive surgical staging of uterine cancer: Gynecologic oncology group study LAP2. Journal of Clinical Oncology, 27, 5331-5336. doi:10.1200/JCO.2009.22.3248

[23] Abu-Abid, S., Szold, A. and Klausner, J. (2002) Obesity and cancer. Journal of Medicine, 33, 73-86.

[24] Palomba, S., Zupi, E., Russo, T., et al. (2007) Presurgical assessment of intraabdominal visceral fat in obese patients with early-stage endometrial cancer treated with laparoscopic approach: Relationships with early laparoscopic conversions. Journal of Minimally Invasive Gynecology, 14, 195-201. doi:10.1016/j.jmig.2006.09.019

[25] Holub, Z., Jabor, A., Sprongl, L. and Fischlova, D. (2002) Clinical outcome, inflammatory response and tissue trauma in total laparoscopic hysterectomy: Comparison to laparoscopically-assisted vaginal hysterectomy. Ces Gynekol, 67, 315-20.

[26] Farthing, A., Chatterjee, J., Joglekar-Pai, P., Dorney, E. and Ghaem-Maghami, S. (2012) Total laparoscopic hysterectomy for early stage endometrial cancer in obese and morbidly obese women. Journal of Obstetrics \& Gynaecology, 32, 580-584. doi:10.3109/01443615.2012.693990

[27] O'Hanlan, K.A., Dibble, S.L. and Fisher, D.T. (2006) Total laparoscopic hysterectomy for uterine pathology: Impact of body mass index on outcomes. Gynecologic Oncology, 103, 938-941. doi:10.1016/j.ygyno.2006.05.036

[28] Yu, C.K., Cutner, A., Mould, T. and Olaitan, A. (2005) Total laparoscopic hysterectomy as a primary surgical treatment for endometrial cancer in morbidly obese women. BJOG, 112, 115-117. doi:10.1111/j.1471-0528.2004.00335.x

[29] Scribner Jr., D.R., Walker, J.L., Johnson, G.A., et al. (2002) Laparoscopic pelvic and para-aotic lymph node dissection in the Obese. Gynecologic Oncology, 84, 426-430. doi:10.1006/gyno.2001.6548

[30] Pavelka, J.C., Ben-Shachar, I., Fowler, J.M., et al. (2004) Morbid obesity and endometrial cancer: Surgical, clinical, and pathologic outcomes in surgically managed patients. Gynecologic Oncology, 95, 588-592. doi:10.1016/j.ygyno.2004.07.047

[31] Obermair, A., Manolitsas, T.P., Yeung, Y., Hammond, I.G. and McCartney, A.J. (2005) Total laparoscopic hysterictomy versus total abdominal hysterectomy for obese wo- men with endometrial cancer. International Journal of Gynecological Cancer, 15, 319-324.

doi:10.1111/j.1525-1438.2005.15223.x

[32] Dumont, L., Mattys, M., Mardirosoff, C., Picard, V., Alle, J.L., et al. (1997) Hemodynamic and pulmonary changes during laparoscopic gastroplasty in morbidly obese patients. Obesity Surgery, 7, 326-331.

[33] O'Gorman, T., MacDonald, N., Mould, T., Cutner, A., Hurley, R. and Olaitan, A. (2009) Total laparoscopic hysterectomy in morbidly obese women with endometrial cancer anaesthetic and surgical considerations. European Journal of Gynaecological Oncology, 30, 171-173

[34] Bloss, J.D., Berman, M.L., Bloss, L.P. and Buller, R.E. (1991) Use of vaginal hysterectomy for the management of stage 1 endometrial cancer in the medically compromised patient. Gynecologic Oncology, 40, 74-77. doi:10.1016/0090-8258(91)90089-N

[35] Childers, J.M. and Surwit, E.A. (1992) Combined laparoscopic and vaginal surgery for the managemement of two cases of stage I endometrial cancer. Gynecologic Oncology, 45, 46-51. doi:10.1016/0090-8258(92)90489-6

[36] Doucette, R.C. and Scott, J.R. (1996) Comparison of laparoscopically assisted vaginal hysterectomy with abdominal and vaginal hysterectomy. Journal of Reproductive Medicine, 41, 1-6.

[37] Sheth, S.S. (2010) Vaginal hysterectomy as a primary route for morbidly obese women. Acta Obstetricia et Gynecologica Scandinavica, 89, 971-974. doi:10.3109/00016341003681256

[38] Guigale, L.E., Di Santo, N., Smolkin, M.E., Havrilesky, L.J. and Modesitt, S.C. (2012) Beyond mere obesity: Effect of increasing obesity classifications on hysterectomy outcomes for uterine cancer/hyperplasia. Gynecologic Oncology, 127, 326-331. doi:10.1016/j.ygyno.2012.08.014

[39] Gehrig, P.A., Cantrell, L.A., Shafer, A., et al. (2008) What is the optimal minimally invasive surgical procedure for endometrial cancer staging in the obese and morbidly obese woman? Gynecologic Oncology, 111, 41-45. doi:10.1016/j.ygyno.2008.06.030

[40] Tang, K.Y., Gardiner, S.K., Gould, C., et al. (2012) Robotic surgical staging for obese women with endometrial cancer. American Journal of Obstetrics \& Gynecology, 206, e1-e6.

[41] Seamon, L.G., Bryant, S.A., Rheaume, P.S., et al. (2009) Comprehensive surgical staging for endometrial cancer in obese patients: Comparing robotics and laparotomy. $\mathrm{Ob}$ stetrics \& Gynecology, 114, 16-21.

[42] Subramaniam, A., Kim, K.H., Bryant, S.A., Zhang, B., et al. (2011) A cohort study evaluating robotic versus laparotomy surgical outcomes of obese women with endometrial cancer. Gynecologic Oncology, 122, 604-607. doi:10.1016/j.ygyno.2011.05.024 